\title{
Ignoring Rotational DoFs in Decoupling Structures Connected Through Flexotorsional Joints
}

\author{
Walter D’Ambrogio* and Annalisa Fregolent** \\ * Dipartimento di Ingegneria Industriale e dell'Informazione e di Economia, Università dell'Aquila \\ Via G. Gronchi, 18 - I-67100, L'Aquila (AQ), Italy, email: walter dambrogio@univaq.it \\ ** Dipartimento di Ingegneria Meccanica e Aerospaziale, Università di Roma La Sapienza \\ Via Eudossiana 18, I 00184 Rome, Italy, email: annalisa.fregolent@uniroma1.it
}

\begin{abstract}
Substructure decoupling consists in the identification of the dynamic behaviour of a structural subsystem, starting from the dynamic behaviour of both the assembled system and the residual subsystem (the known portion of the assembled system). The degrees of freedom (DoFs) of the coupled system can be partitioned into internal DoFs (not belonging to the couplings) and coupling DoFs. In direct decoupling, a fictitious subsystem that is the negative of the residual subsystem is added to the coupled system, and appropriate compatibility and equilibrium conditions are enforced at interface DoFs. Compatibility and equilibrium can be required either at coupling DoFs only (standard interface), or at additional internal DoFs of the residual subsystem (extended interface), or at some coupling DoFs and some internal DoFs of the residual subsystem (mixed interface). In this paper, a test bench is considered made by a cantilever column with two staggered short arms coupled to a horizontal beam. This involves both flexural and torsional DoFs, on which rotational FRFs are quite difficult to measure. Using a mixed interface, rotational DoFs are neglected and substituted by internal translational DoFs. Experimental results are presented and discussed.
\end{abstract}

Keywords: Substructure Decoupling, Rotational DoFs, Flexotorsional Joints, Mixed Interface, Experimental Dynamic Substructuring.

\section{INTRODUCTION}

Substructure decoupling consists in the identification of the dynamic behaviour of a structural subsystem, starting from the dynamic behaviour of both the assembled system and the residual subsystem (the known portion of the assembled system). Decoupling is a need for subsystems that cannot be measured separately, but only when coupled to their neighboring substructure(s) (e.g. fixtures needed for testing or subsystems in operational conditions).

Substructure decoupling represents a special case of experimental dynamic substructuring, where experimental means that the model of at least one subsystem derives from tests. In Frequency Based Substructuring, Frequency Response Functions (FRFs) are used instead of modal parameters to avoid modal truncation problems. A general framework for dynamic substructuring is provided in [1], where primal and dual assembly are introduced.

A well known issue in experimental dynamic substructuring is related to rotational DoFs. In substructure coupling, whenever coupling DoFs include rotational DoFs, the related rotational FRFs must be obtained experimentally. This 
becomes a quite complicated task when measuring only translational FRFs, as shown in [2]. Several techniques for measuring rotational responses have been devised since then, see e.g. [3, 4]. However, when such rotational FRFs are used for substructure coupling, results are still unsatisfactory.

Substructure decoupling techniques can be classified as reverse coupling techniques or direct decoupling techniques. In reverse coupling, the equations written for the coupling problem are rearranged to isolate (as unknown) one of the substructures instead of the assembled structure. Examples of reverse coupling are impedance and mobility approaches $[5,6]$.

In direct decoupling, a fictitious subsystem that is the negative of the residual subsystem is added to the coupled system, and appropriate compatibility and equilibrium conditions are enforced at interface DoFs. To solve the decoupling problem, a dual assembly [7], a primal assembly [8] or an hybrid assembly $[9,10]$ can be used. Compatibility and equilibrium can be required either at coupling DoFs only (standard interface), or at additional internal DoFs of the residual subsystem (extended interface), or at subsets of coupling DoFs and internal DoFs of the residual subsystem (mixed interface). The choice of interface DoFs determines a set of frequencies at which the decoupling problem is ill conditioned, as shown in [7].

For some time, it was believed that issues related to rotational DoFs also applied to substructure decoupling. However, in this case the actions exchanged through the connecting DoFs, and specifically through rotational DoFs, are already embedded in each FRF of the assembled system. In practice, a mixed interface [11] can in fact be considered that allows to substitute undesired coupling DoFs with internal DoFs of the residual subsystem. This approach is introduced in [12] using simulated test data.

In this paper, a test bed is considered made by a cantilever column with two staggered short arms coupled to a horizontal beam. This involves both flexural and torsional DoFs, on which rotational FRFs are quite difficult to measure. Using a mixed interface, rotational DoFs are neglected and substituted by internal translational DoFs. Experimental results are presented and discussed.

\section{DIRECT DECOUPLING USING DUAL ASSEMBLY}

The unknown substructure $U\left(N_{U}\right.$ DoFs) is a portion of a larger structure $R U$ ( $N_{R U}$ DoFs). The known portion of the assembled structure $R U$, defined as residual substructure $R\left(N_{R} \mathrm{DoFs}\right)$, is joined to the unknown substructure through a number of couplings (see Fig. 1). The degrees of freedom (DoFs) can be partitioned into internal DoFs (not belonging to the couplings) of substructure $U(u)$, internal DoFs of substructure $R(r)$, and coupling DoFs $(c)$.

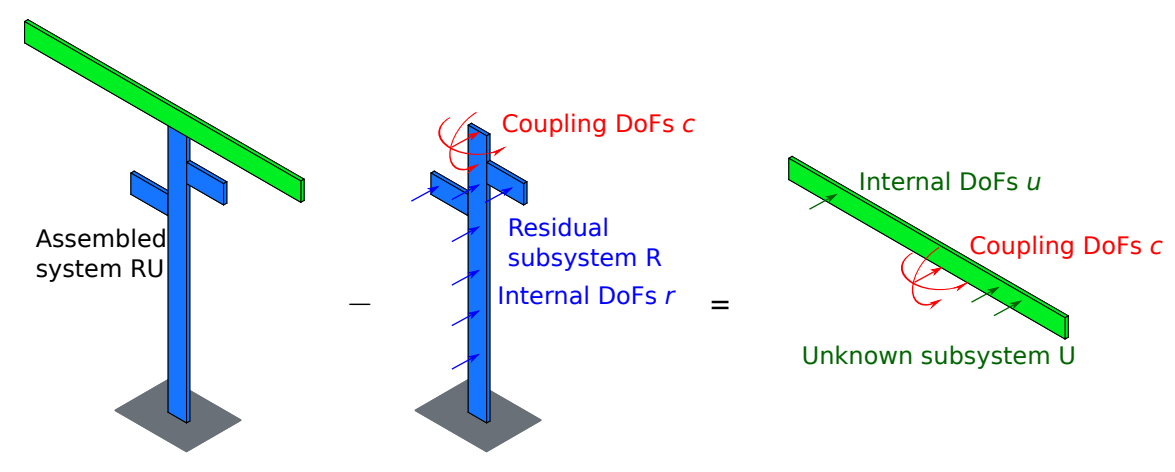

Figure 1: Scheme of the direct decoupling problem.

The goal is to find the FRF of the unknown substructure $U$ starting from the FRFs of the assembled structure $R U$ and of the residual substructure $R$. The dynamic behaviour of the unknown substructure $U$ can be extracted 
from that of the assembled structure $R U$ by taking out the dynamic effect of the residual subsystem $R$. This can be accomplished by considering a negative structure, i.e. by adding to the assembled structure $R U$ a fictitious substructure with a dynamic stiffness opposite to that of the residual substructure $R$ and satisfying compatibility and equilibrium conditions. The dynamic equilibrium of the assembled structure $R U$ and of the negative substructure is expressed in block diagonal format as:

$$
\left[\begin{array}{cc}
Z^{R U} & 0 \\
0 & -Z^{R}
\end{array}\right]\left\{\begin{array}{c}
u^{R U} \\
u^{R}
\end{array}\right\}=\left\{\begin{array}{c}
f^{R U} \\
f^{R}
\end{array}\right\}+\left\{\begin{array}{c}
g^{R U} \\
g^{R}
\end{array}\right\}
$$

where:

- $\mathbf{Z}^{\mathrm{RU}},-\mathbf{Z}^{\mathrm{R}}$ are the dynamic stiffness matrices of the assembled structure $R U$ and of the negative structure, respectively;

- $\mathbf{u}^{\mathbf{R U}}, \mathbf{u}^{\mathbf{R}}$ are the vectors of degrees of freedom of the assembled structure $R U$ and of the negative structure, respectively;

- $\mathbf{f}^{\mathrm{RU}}, \mathbf{f}^{\mathbf{R}}$ are the external force vectors on the assembled structure $R U$ and on the negative structure, respectively;

- $\mathbf{g}^{\mathbf{R U}}, \mathbf{g}^{\mathbf{R}}$ are the vectors of disconnection forces exchanged between the assembled structure and the negative structure (constraint forces associated with compatibility conditions).

Compatibility and equilibrium conditions must be considered at the interface between the assembled structure $R U$ and the negative structure: such interface includes not only all the coupling DoFs between substructures $U$ and $R$, but includes as well all the internal DoFs of substructure $R$ (the blue part of the structure in Fig. 1). However, it is not required to consider all these interface DoFs, because it is sufficient that the number of interface DoFs be not less than the number of coupling DoFs $n_{c}$. Therefore, several options for interface DoFs can be considered:

- standard interface, including only the coupling DoFs $(c)$ between substructures $U$ and $R$;

- extended interface, including also a subset of internal DoFs $(i \subseteq r)$ of the residual substructure $R$;

- mixed interface, including subsets of coupling DoFs $(d \subseteq c)$ and/or internal DoFs $(i \subseteq r)$ of substructure $R$.

The use of a mixed interface may allow to ignore rotational coupling DoFs by substituting them with translational internal DoFs.

The compatibility condition at the (standard, extended, mixed) interface DoFs implies that any pair of matching DoFs $u_{l}^{R U}$ and $u_{m}^{R}$, i.e. DoF I on the coupled system $R U$ and DoF $m$ on subsystem $R$ must have the same displacement, that is $u_{1}^{R U}-u_{m}^{R}=0$. Let the number of interface DoFs on which compatibility is enforced be denoted as $N_{C}$.

The compatibility condition can be generally expressed as:

$$
\left[\begin{array}{ll}
\mathbf{B}_{\mathrm{C}}^{\mathrm{RU}} & \mathbf{B}_{\mathrm{C}}^{\mathbf{R}}
\end{array}\right]\left\{\begin{array}{c}
\mathbf{u}^{\mathrm{RU}} \\
\mathbf{u}^{\mathbf{R}}
\end{array}\right\}=0
$$

where each row of $\mathbf{B}_{\mathbf{C}}=\left[\mathbf{B}_{\mathbf{C}}^{\mathbf{R U}} \mathbf{B}_{\mathbf{C}}^{\mathbf{R}}\right]$ corresponds to a pair of matching DoFs. Note that $\mathbf{B}_{\mathbf{C}}$ has size $N_{C} \times\left(N_{R U}+N_{R}\right)$ and is, in most cases, a signed Boolean matrix. 
It should be noted that the interface DoFs involved in the equilibrium condition need not to be the same used to enforce the compatibility condition, as long as compatibility can be ensured by disconnection forces applied at a different set of DoFs. If the compatibility and the equilibrium DoFs are not the same, the approach is called noncollocated [11]. Obviously, the traditional approach, in which compatibility and equilibrium DoFs are the same, is called collocated.

Let $N_{E}$ denote the number of interface DoFs on which equilibrium is enforced. The equilibrium of disconnection forces implies that their sum must be zero for any pair of matching DoFs belonging to the equilibrium interface, i.e. $g_{r}^{R U}+g_{s}^{R}=0$. Furthermore, for any DoF $k$ on the coupled system $R U$ (or on the residual subsystem $R$ ) not belonging to the equilibrium interface, it must be $g_{k}^{R U}=0\left(g_{k}^{R}=0\right)$.

Overall, the above conditions can be expressed as:

$$
\left[\begin{array}{c}
\mathrm{L}_{\mathrm{E}}^{\mathrm{RU}} \\
\mathrm{L}_{\mathrm{E}}^{\mathrm{R}}
\end{array}\right]^{T}\left\{\begin{array}{c}
\mathbf{g}^{\mathrm{RU}} \\
\mathbf{g}^{\mathrm{R}}
\end{array}\right\}=0
$$

where the matrix $L_{E}=\left[L_{E}^{R U} L_{E}^{R}\right]$ is a Boolean localisation matrix. Note that the number of columns of $L_{E}$ is equal to the number $N_{E}$ of equilibrium interface DoFs plus the number $N_{N E}$ of DoFs not belonging to the equilibrium interface. Note that $N_{N E}=N_{R U}+N_{R}-2 N_{E}$ : in fact, the number of DoFs belonging to the equilibrium interface must be subtracted once from $N_{R U}$ and once from $N_{R}$. Therefore, the size of $L_{E}$ is $\left(N_{R U}+N_{R}\right) \times\left(N_{R U}+N_{R}-N_{E}\right)$.

Eqs. (1-3) can be gathered to obtain the so-called 3-field formulation. Starting from the 3-field formulation, several assembly techniques can be devised:

- dual assembly $[1,7]$ where equilibrium is satisfied exactly by defining a unique set of disconnection force intensities;

- primal assembly $[1,8]$ where compatibility is satisfied exactly by defining a unique set of interface DoFs;

- hybrid assembly $[9,10]$ where both compatibility and equilibrium are satisfied exactly.

In the sequel, only the dual assembly is recalled. It can be shown [9] that whenever $N_{C}=N_{E}$, i.e. the number of compatibility DoFs is the same as the number of equilibrium DoFs, all assembly techniques provide the same result.

\subsection{Dual assembly}

In the dual assembly, the equilibrium condition $g_{r}^{R U}+g_{s}^{R}=0$ at a pair of equilibrium interface DoFs is ensured by choosing $g_{r}^{R U}=-\lambda$ and $g_{s}^{R}=\lambda$. If a Boolean matrix $\mathbf{B}_{\mathrm{E}}$ related to interface equilibrium DoFs is defined similarly to $\mathbf{B}_{\mathbf{C}}$, the overall interface equilibrium can be ensured by writing the disconnection forces in the form:

$$
\left\{\begin{array}{c}
\mathbf{g}^{\mathrm{RU}} \\
\mathbf{g}^{\mathbf{R}}
\end{array}\right\}=-\left[\begin{array}{c}
\mathbf{B}_{\mathrm{E}}^{\mathrm{RU}^{T}} \\
\mathbf{B}_{\mathrm{E}}^{\mathbf{R}^{T}}
\end{array}\right] \boldsymbol{\lambda}
$$

where $\lambda$ are Lagrange multipliers corresponding to disconnection force intensities and $\mathbf{B}_{\mathrm{E}}$ is a $N_{E} \times\left(N_{R U}+N_{R}\right)$ matrix. Since there is a unique set of disconnection force intensities $\lambda$, the interface equilibrium condition is satisfied automatically for any $\lambda$, i.e. 


$$
\left[\begin{array}{c}
\mathrm{L}_{\mathrm{E}}^{\mathrm{RU}} \\
\mathrm{L}_{\mathrm{E}}^{\mathrm{R}}
\end{array}\right]^{T}\left\{\begin{array}{c}
\mathbf{g}^{\mathrm{RU}} \\
\mathbf{g}^{\mathbf{R}}
\end{array}\right\}=-\left[\begin{array}{c}
\mathrm{L}_{\mathrm{E}}^{\mathrm{RU}} \\
\mathrm{L}_{\mathrm{E}}^{\mathrm{R}}
\end{array}\right]^{T}\left[\begin{array}{c}
\mathbf{B}_{\mathrm{E}}^{\mathrm{RU}}{ }^{T} \\
\mathbf{B}_{\mathrm{E}}^{\mathbf{R}^{T}}
\end{array}\right] \boldsymbol{\lambda}=0
$$

In the dual assembly, the total set of DoFs is retained, i.e. each interface DoF appears twice. Since Eq. (5) is always satisfied, the 3-field formulation reduces to:

$$
\left\{\begin{array}{l}
{\left[\begin{array}{cc}
\mathbf{Z}^{\mathbf{R U}} & 0 \\
0 & -\mathbf{Z}^{\mathbf{R}}
\end{array}\right]\left\{\begin{array}{l}
\mathbf{u}^{\mathbf{R U}} \\
\mathbf{u}^{\mathbf{R}}
\end{array}\right\}+\left[\begin{array}{c}
\mathbf{B}_{\mathrm{E}}^{\mathbf{R U}^{T}} \\
\mathbf{B}_{\mathrm{E}}^{\mathbf{R}^{T}}
\end{array}\right] \lambda=\left\{\begin{array}{c}
\mathbf{f}^{\mathbf{R U}} \\
\mathbf{f}^{\mathbf{R}}
\end{array}\right\}} \\
{\left[\begin{array}{ll}
\mathbf{B}_{\mathbf{C}}^{\mathrm{RU}} & \mathbf{B}_{\mathbf{C}}^{\mathbf{R}}
\end{array}\right]\left\{\begin{array}{l}
\mathbf{u}^{\mathbf{R U}} \\
\mathbf{u}^{\mathbf{R}}
\end{array}\right\}=0}
\end{array}\right.
$$

or in more compact form:

$$
\left\{\begin{array}{l}
\mathbf{Z} \mathbf{u}+\mathbf{B}_{\mathbf{E}}^{T} \lambda=\mathbf{f} \\
\mathbf{B}_{\mathbf{C}} \mathbf{u}=0
\end{array}\right.
$$

To eliminate $\lambda$, Eq. $\left(1^{*}\right)$ can be written:

$$
\mathbf{u}=-\mathbf{Z}^{-1} \mathbf{B}_{\mathbf{E}}^{T} \lambda+\mathbf{Z}^{-1} \mathbf{f}
$$

which substituted in Eq. (2) gives:

$$
\mathbf{B}_{\mathbf{C}} \mathbf{Z}^{-1} \mathbf{B}_{\mathbf{E}}^{T} \lambda=\mathbf{B}_{\mathbf{C}} \mathbf{Z}^{-1} \mathbf{f}
$$

from which $\lambda$, to be back-substituted in Eq. $\left(1^{*}\right)$, is found as:

$$
\lambda=\left(\mathbf{B}_{\mathbf{C}} \mathbf{Z}^{-1} \mathbf{B}_{\mathbf{E}}^{T}\right)^{+} \mathbf{B}_{\mathbf{C}} \mathbf{Z}^{-1} \mathbf{f}
$$

To obtain a determined or overdetermined matrix for the generalized inversion operation, the number of rows of $\mathbf{B}_{\mathbf{C}}$ must be greater or equal than the number of rows of $B_{E}$, i.e.

$$
N_{C} \geq N_{E} \geq n_{C}
$$

Note that, if $N_{C}>N_{E}$, Eq. (6) is not satisfied exactly by vector $\lambda$ given by Eq. (7), but only in the minimum square sense. This implies that also Eq. (2) is not satisfied exactly, i.e. compatibility conditions at interface are approximately satisfied. On the contrary, equilibrium is satisfied exactly due to the introduction of the disconnection force intensities $\lambda$ as in Eq. (4). 
By substituting $\lambda$ in Eq. $\left(1^{*}\right)$, it is obtained:

$$
\mathbf{Z u}+\mathbf{B}_{\mathrm{E}}^{T}\left(\mathbf{B}_{\mathrm{C}} \mathbf{Z}^{-1} \mathbf{B}_{\mathrm{E}}^{T}\right)^{+} \mathbf{B}_{\mathrm{C}} \mathbf{Z}^{-1} \mathbf{f}=\mathbf{f}
$$

Finally, $\mathbf{u}$ can be written as $\mathbf{u}=\mathbf{H f}$, which provides the FRF of the unknown subsystem $\mathbf{U}$ :

$$
\mathbf{u}=\left(\mathbf{Z}^{-1}-\mathbf{Z}^{-1} \mathbf{B}_{\mathrm{E}}^{T}\left(\mathbf{B}_{\mathrm{C}} \mathbf{Z}^{-1} \mathbf{B}_{\mathrm{E}}^{T}\right)^{+} \mathbf{B}_{\mathrm{C}} \mathbf{Z}^{-1}\right) \mathbf{f}
$$

i.e., by noting that the inverse of the block diagonal dynamic stiffness matrix can be expressed as:

$$
\left[\begin{array}{cc}
\mathbf{Z}^{\mathrm{RU}} & 0 \\
0 & -\mathbf{Z}^{\mathrm{R}}
\end{array}\right]=\mathbf{Z}^{-1}=\mathbf{H}=\left[\begin{array}{cc}
\mathbf{H}^{\mathrm{RU}} & 0 \\
0 & -\mathbf{H}^{\mathbf{R}}
\end{array}\right]
$$

where $\mathbf{H}^{\mathbf{R U}}$ and $\mathbf{H}^{\mathbf{R}}$ are the FRFs of the assembled structure and of the residual substructure, it is:

$$
\mathbf{H}^{\mathrm{U}}=\mathbf{H}-\mathrm{HB}_{\mathrm{E}}^{T}\left(\mathbf{B}_{\mathrm{C}} \mathrm{HB}_{\mathrm{E}}{ }^{T}\right)^{+} \mathbf{B}_{\mathrm{C}} \mathbf{H}
$$

With the dual assembly, the rows and the columns of $\mathbf{H}^{\mathrm{U}}$ corresponding to compatibility and equilibrium DoFs appear twice. Furthermore, when using an extended or mixed interface, $\mathbf{H}^{\mathrm{U}}$ contains some meaningless rows and columns: those corresponding to the internal DoFs of the residual substructure $R$. Obviously, only meaningful and independent entries are retained.

\subsection{Interface flexibility matrix}

In Eq. (12), the product of the three matrices to be inverted can be defined as interface flexibility matrix. The interface flexibility matrix can be rewritten in expanded form as:

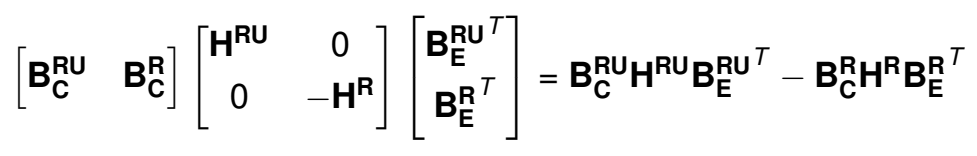

It can be noticed that

$$
\mathrm{B}_{\mathrm{C}}^{\mathrm{RU}} \mathrm{H}^{\mathrm{RU}} \mathrm{B}_{\mathrm{E}}^{\mathrm{RU}}=\hat{\mathrm{H}}^{\mathrm{RU}}
$$

where $\hat{H}^{\mathrm{RU}}$ is a subset of the FRF matrix of the coupled structure: pre-multiplication by $\mathbf{B}_{C}^{\mathrm{RU}}$ extracts rows at compatibility DoFs, and post-multiplication by $\mathbf{B}_{\mathrm{E}}^{\mathrm{RU}}$ extracts columns at the equilibrium DoFs. Similarly,

$$
\mathbf{B}_{C}^{\mathbf{R}} \mathbf{H}^{\mathbf{R}} \mathbf{B}_{\mathrm{E}}^{\mathbf{R}^{T}}=\hat{\mathbf{H}}^{\mathbf{R}}
$$

where $\hat{\mathbf{H}}^{\mathbf{R}}$ is the FRF of the residual structure at the same DoFs as above. 


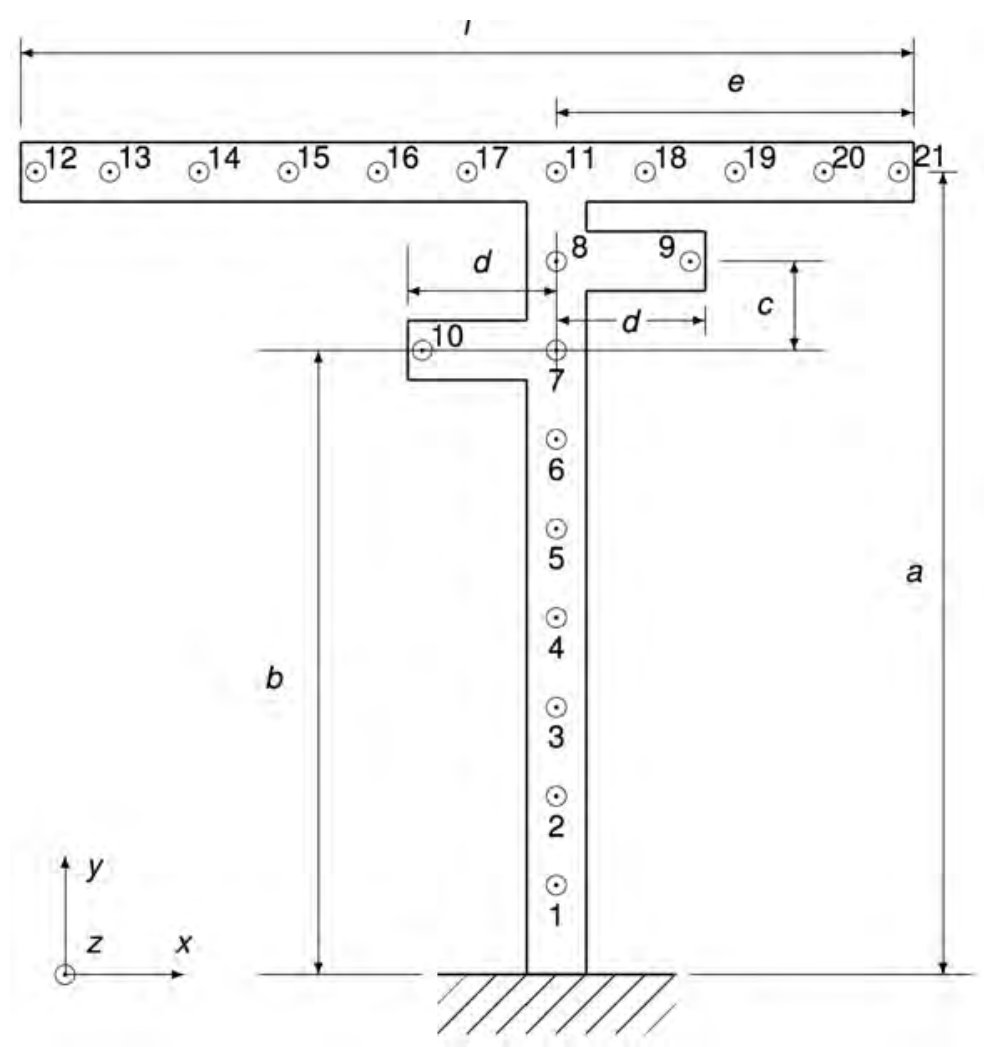

Figure 2: Sketch of the test structure.

Therefore, the interface flexibility matrix becomes:

$$
\mathbf{B}_{\mathrm{C}}^{\mathrm{RU}} \mathrm{H}^{\mathrm{RU}} \mathbf{B}_{\mathrm{E}}^{\mathrm{RU}}{ }^{T}-\mathbf{B}_{\mathrm{C}}^{\mathrm{R}} \mathrm{H}^{\mathrm{R}} \mathbf{B}_{\mathrm{E}}^{\mathbf{R}^{T}}=\hat{\mathbf{H}}^{\mathrm{RU}}-\hat{\mathbf{H}}^{\mathbf{R}}
$$

The interface flexibility matrix (14) is strictly related to singularity. In $[7,8]$, it is shown that the interface flexibility matrix is singular at the resonant frequencies of the residual substructure with coupling DoFs grounded, both for standard interface and for non collocated extended interface when equilibrium condition is enforced on coupling DoFs only. In other cases, it is not so easy to find the frequencies at which the interface flexibility matrix is singular.

\section{$3 \quad$ TEST STRUCTURE}

The proposed decoupling technique is tested on an aluminium structure (Fig. 2). The residual substructure $R$ consists of a cantilever column with two staggered short arms. The unknown substructure $U$ is a horizontal beam. The horizontal beam is bolted to the top of the column, involving both translational and rotational DoFs.

The geometrical dimensions are reported in Table 1 . The cross section is $40 \mathrm{~mm} \times 8 \mathrm{~mm}$ for all beams, with the short side along the $z$-direction.

\begin{tabular}{cccccc}
\hline$a$ & $b$ & $c$ & $d$ & $e$ & $l$ \\
\hline 540 & 420 & 60 & 100 & 240 & 600 \\
\hline
\end{tabular}

TABLE 1: Geometrical dimensions [mm] 


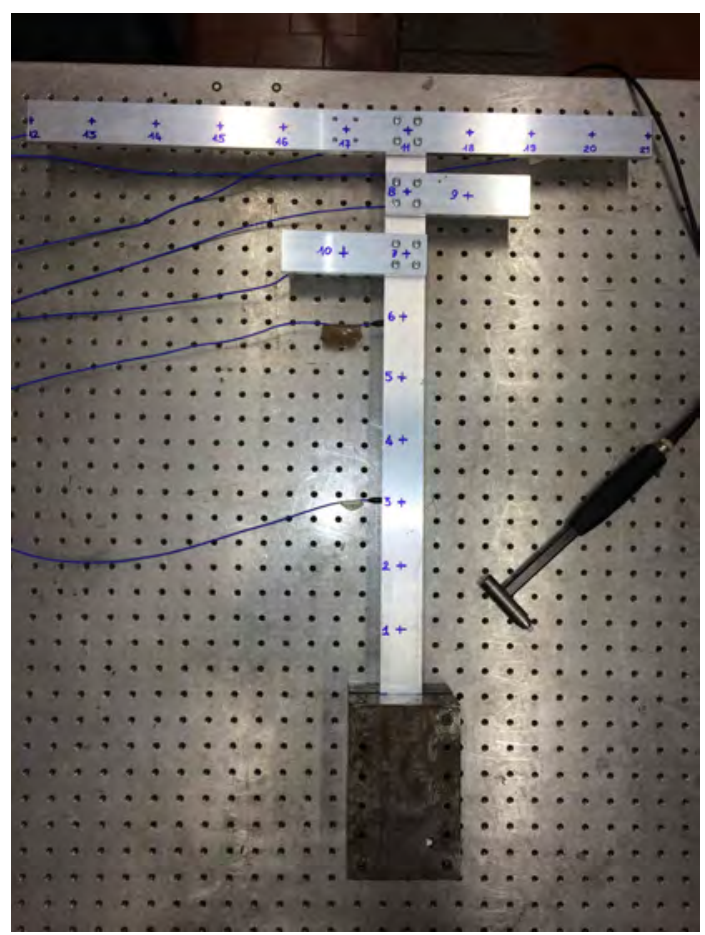

Figure 3: Assembled system.

The experimental FRFs of the assembled system $R U$ up to $2000 \mathrm{~Hz}$ are obtained by applying impact excitation and measuring the resulting accelerations along $z$-direction at seven locations $(3,6,9,10,11,13,20)$, as shown in Fig. 3. For the residual subsystem $R$ (column) the experimental FRFs are similarly measured at five locations $(3,6,9,10,11)$, as shown in Fig. 4. A detail of the bolted junction between the beam and the column is shown in Fig. 5. Finally, to check decoupling results, FRFs are measured also at three locations $(11,13,20)$ of the unknown subsystem $U$ (beam), supported by an inflated rubber tube, shown in Fig. 6, giving rigid body eigenfrequencies well separated from the first flexible mode of the beam. Measurements are performed by placing the accelerometers at the underside of each (sub)structure. In order to obtain a complete FRF matrix, as required by the decoupling technique, impact excitation is sequentially provided on all DoFs at the topside of each (sub)structure.

A reciprocity check is performed on the experimental FRFs showing that reciprocity is acceptable for all FRF pairs involving coupling DoFs and internal DoFs of the residual subsystem, i.e. the DoFs that can be used to enforce compatibility and equilibrium conditions. Figures 7 and 8 show the reciprocity check on experimental FRFs of the assembled structure $R U$ and of the residual subsystem $R$. No indication about possible FRFs that should be discarded because of lack of reciprocity is provided by this check.

\section{DECOUPLING}

The FRFs of subsystem $U$ can be determined through the procedure described previously and summarized in Eq. (12), where compatibility and equilibrium DoFs are defined case by case. A collocated approach is adopted in which compatibility and equilibrium DoFs are the same.

FRFs to be used in decoupling can be either the raw FRFs or can be obtained by a curve fitting procedure. In the latter case, results are very bad and are not shown in the paper. This occurs although curve fitting is performed using a global procedure that gives rise to a unique modal model plus low and high frequency residuals obtained for all FRFs. Therefore, raw FRFs are used in the sequel. 


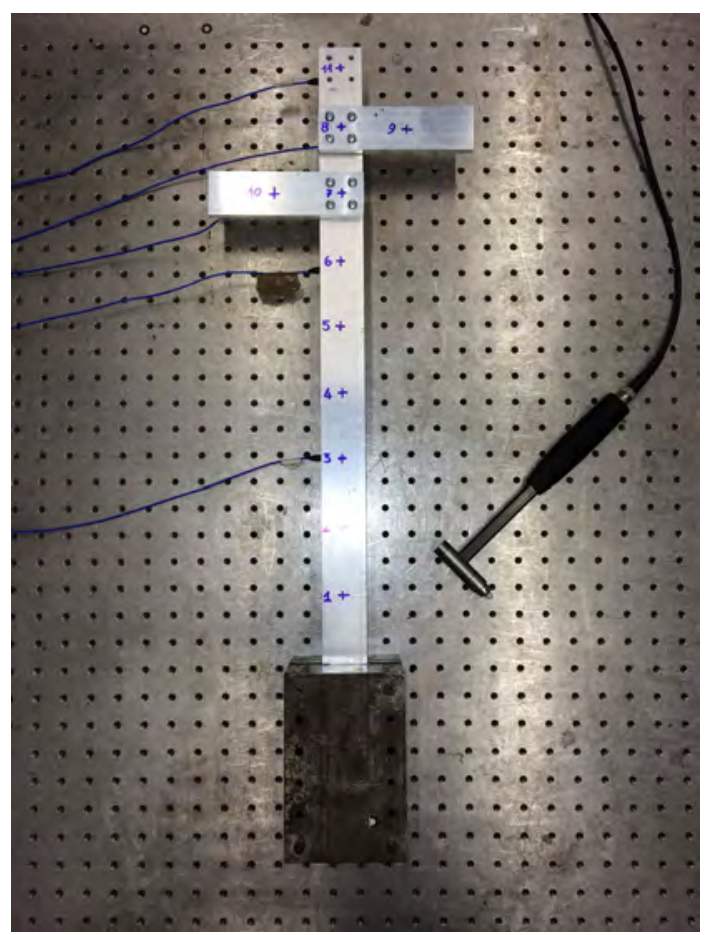

Figure 4: Residual subsystem.

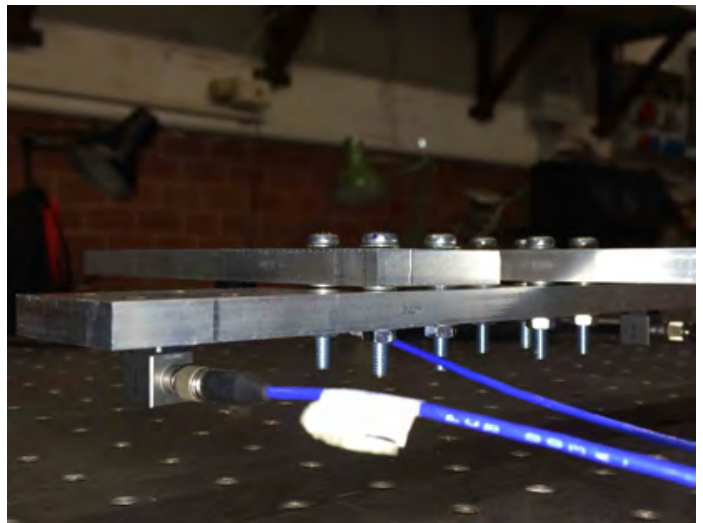

Figure 5: Detail of the bolted junction.

\subsection{Results using raw FRFs}

Since rotational DoFs at the junction between the residual subsystem and the unknown subsystem can not be measured, neither the standard interface nor the extended interface can be used. Therefore, only mixed interfaces can be considered. The number $n_{c}$ of coupling DoFs is 3 so that it must be $N_{E} \geq n_{c}=3$. To deal with overdetermined problems, a set of attempts using mixed interfaces with $N_{C}=N_{E}=4$ is performed. 


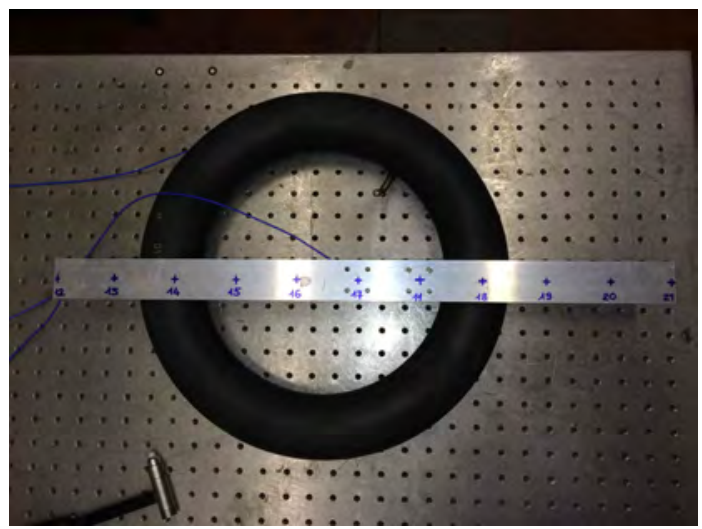

Figure 6: Unknown subsystem.
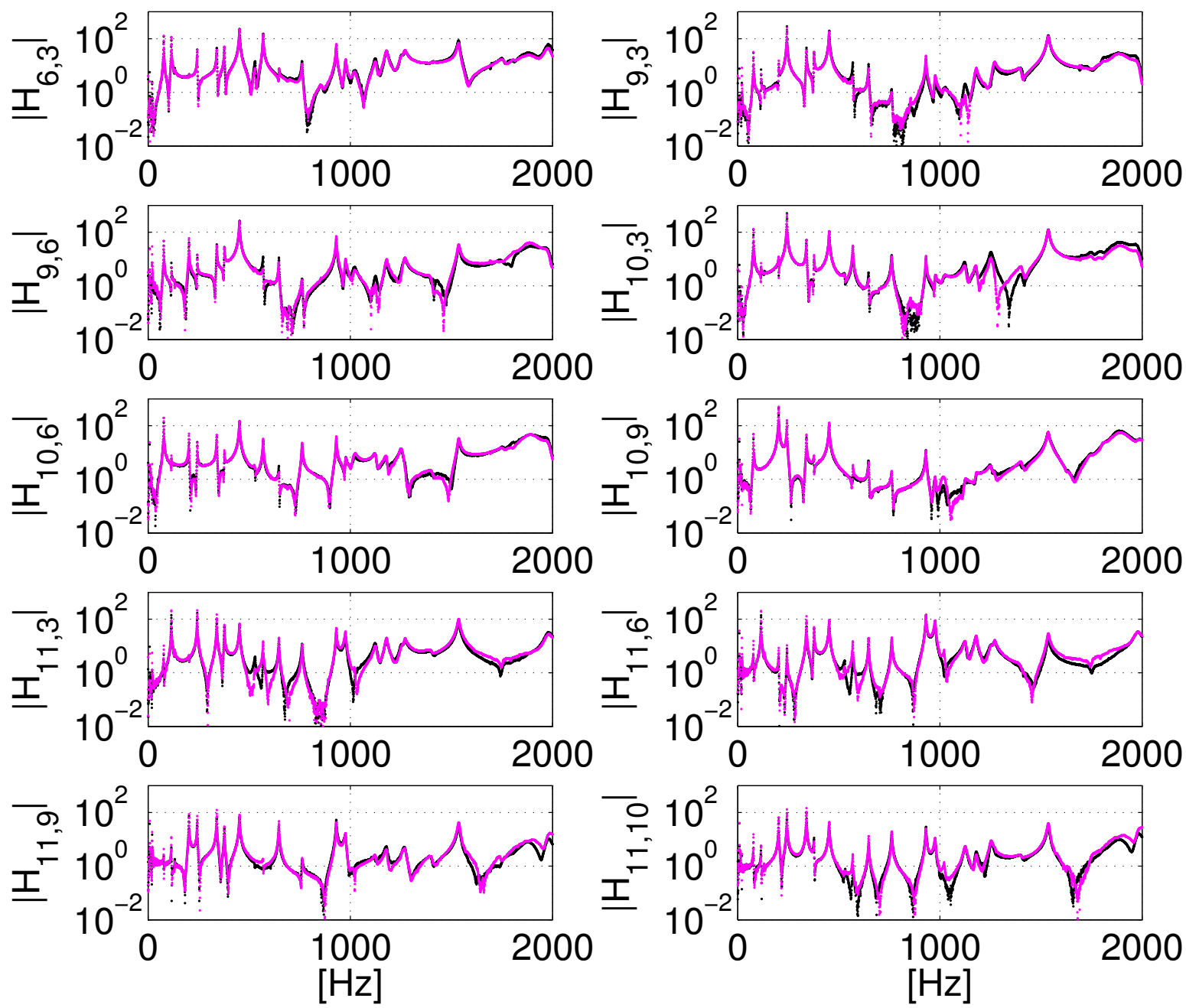

Figure 7: Reciprocity check on experimental FRFs of the assembled structure $R U$ among DoFs 3, 6, 9, $10,11$. 

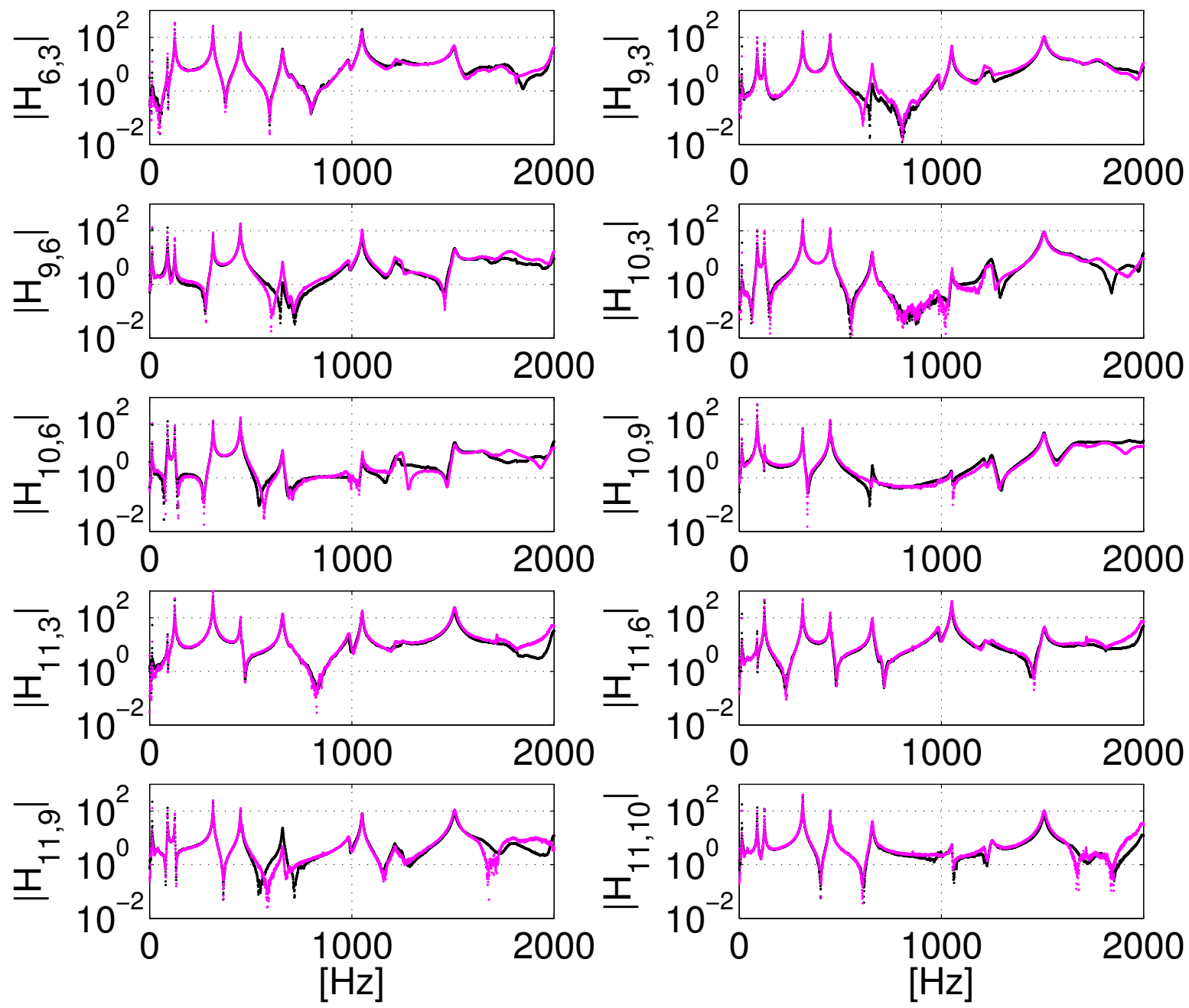

Figure 8: Reciprocity check on experimental FRFs of the residual structure $R U$ among DoFs 3, 6, 9, $10,11$.

First, an interface including DoFs $3 z, 6 z, 9 z$ and $11 z$ is used. Therefore

$$
\mathbf{B}_{\mathbf{C}}=\mathbf{B}_{\mathbf{E}}=\left[\begin{array}{cccc|rrrr}
u_{3 z}^{R U} & u_{6 z}^{R U} & u_{9 y}^{R U} & u_{11 z}^{R U} & u_{3 z}^{R} & u_{6 z}^{R} & u_{9 z}^{R} & u_{11 z}^{R} \\
1 & 0 & 0 & 0 & -1 & 0 & 0 & 0 \\
0 & 1 & 0 & 0 & 0 & -1 & 0 & 0 \\
0 & 0 & 1 & 0 & 0 & 0 & -1 & 0 \\
0 & 0 & 0 & 1 & 0 & 0 & 0 & -1
\end{array}\right]
$$

The FRF of the unknown substructure $U$ is shown in Fig. 9. It can be noticed that, although the FRF is not very scattered, the peak around $1000 \mathrm{~Hz}$ is not well described and some other peaks are shifted towards higher frequencies.

Another mixed interface including DoFs $3 z, 6 z, 10 z$ and $11 z$ is used. The signed Boolean matrices $\mathbf{B}_{\mathrm{C}}$ and $\mathbf{B}_{\mathrm{E}}$ are built as in the previous case. The FRF of the unknown substructure $U$ is shown in Fig. 10. It can be noticed 


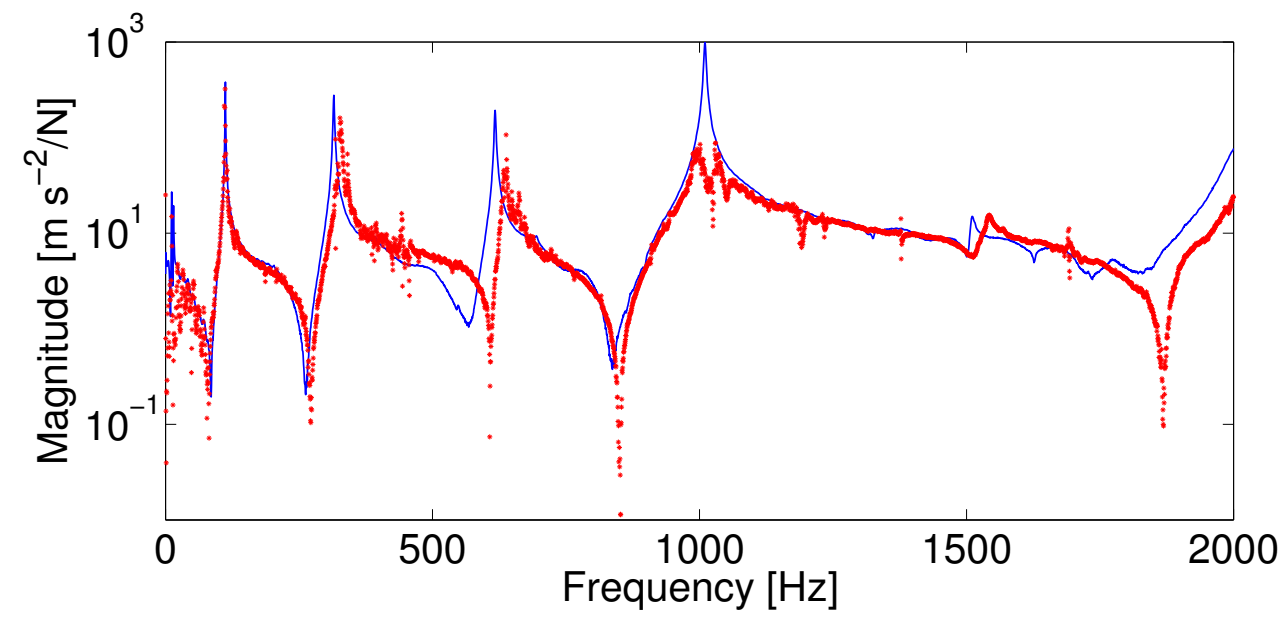

Figure 9: $H_{11 z, 11 z}^{U}$ : measured $(-)$, computed using mixed interface with coupling DoF 11z, and internal DoFs $3 z, 6 z, 9 z(* * *)$.

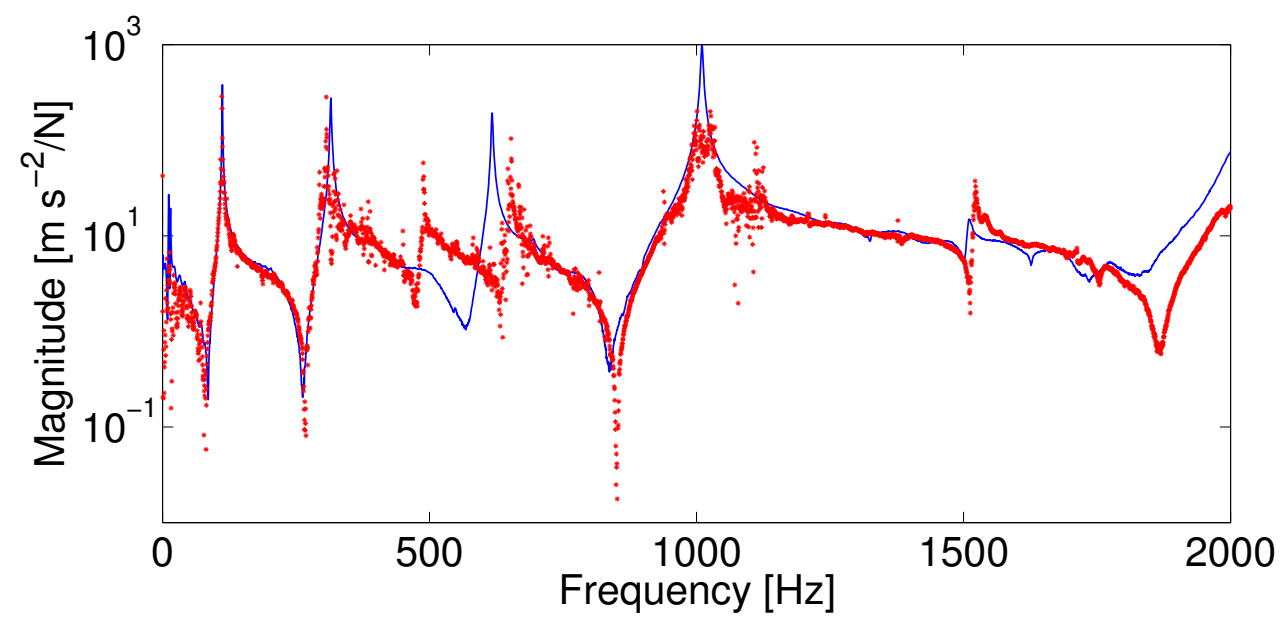

Figure 10: $H_{11 z, 11 z}^{U}$ : measured (-), computed using mixed interface with coupling DoF 11z, and internal DoFs $3 z, 6 z, 10 z(* * *)$.

that around $500 \mathrm{~Hz}$ a spurious peak appear and another peak is considerably forward shifted. However, the peak around $1000 \mathrm{~Hz}$ is better described.

Subsequently, a mixed interface including DoFs $3 z, 9 z, 10 z$ and $11 z$ is used. The signed Boolean matrices $\mathbf{B}_{\mathbf{C}}$ and $\mathbf{B}_{\mathrm{E}}$ are built as in the first case. The FRF of the unknown substructure $U$ is shown in Fig. 11. It can be noticed that around $600 \mathrm{~Hz}$ the peak is shifted forward and around this peak the FRF is a bit scattered. However, the peak around $1000 \mathrm{~Hz}$ is very well described.

Finally, a mixed interface including DoFs $6 z, 9 z, 10 z$ and $11 z$ is used. The signed Boolean matrices $\mathbf{B}_{\mathrm{C}}$ and $\mathbf{B}_{\mathrm{E}}$ are built as in the first case. The FRF of the unknown substructure $U$ is shown in Fig. 12. At a first glance, the result looks very similar to the previous one (interface DoFs $3 z, 9 z, 10 z$ and $11 z$ ), but it is definitely worse because of several spikes and because the FRF around $1000 \mathrm{~Hz}$ is described less accurately than in the previous case.

A new set of attempts is performed using mixed interfaces including only 3 DoFs that give rise to determined problems. Based on the previous results, a mixed interface that includes DoFs $3 z, 9 z$ and $11 z$ is used. Such DoFs represent the set intersection between DoFs $3 z, 6 z, 9 z, 11 z$ and $3 z, 9 z, 10 z, 11 z$ that provide the best results 


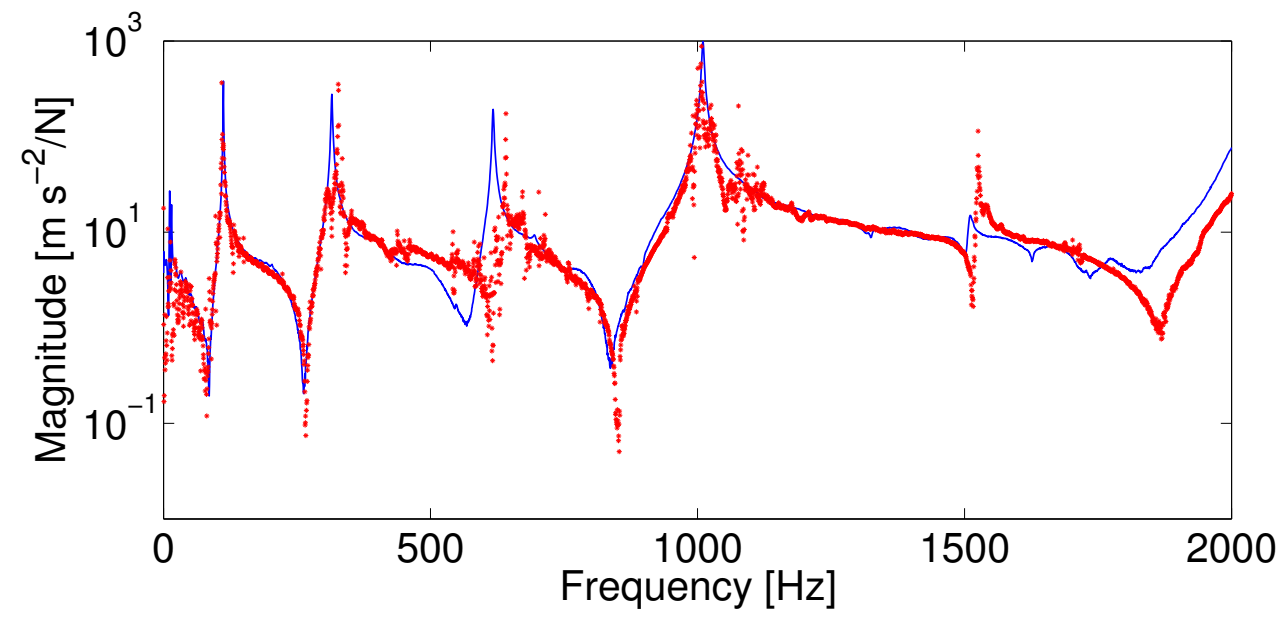

Figure 11: $H_{11 z, 11 z}^{U}$ : measured $(-)$, computed using mixed interface with coupling DoF 11z, and internal DoFs $3 z, 9 z, 10 z(* * *)$.

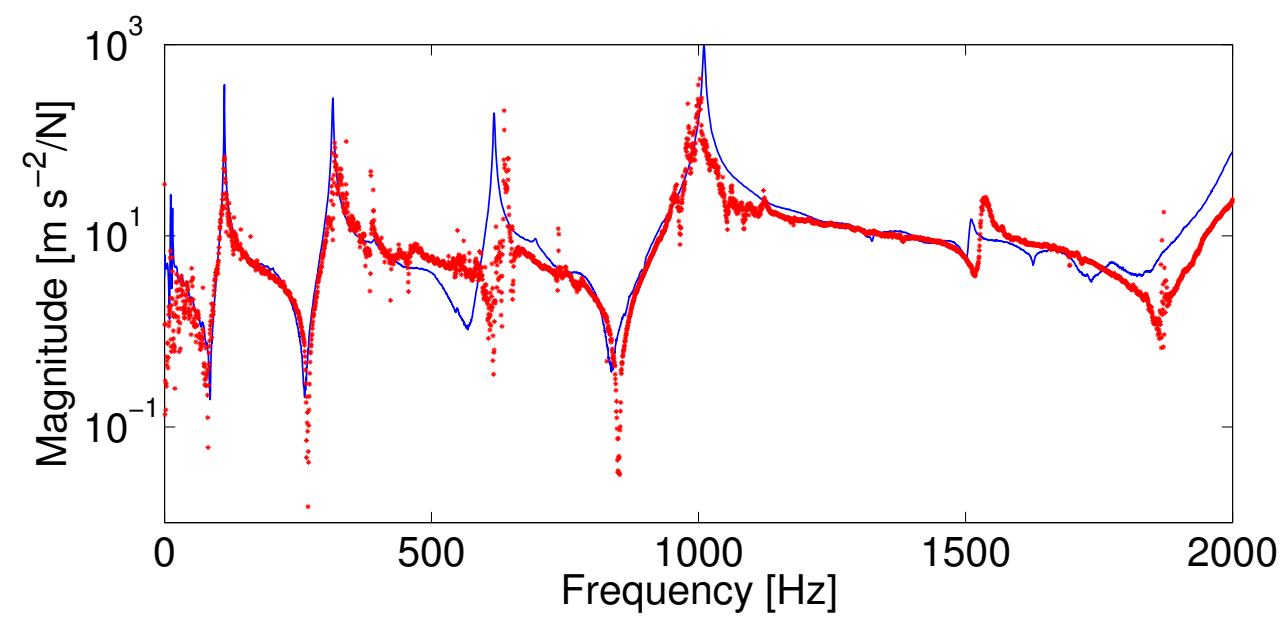

Figure 12: $H_{11 z, 11 z}^{U}$ : measured (-), computed using mixed interface with coupling DoF 11z, and internal DoFs $6 z, 9 z, 10 z(* *)$.

using 4 interface DoFs. The signed Boolean matrices $\mathbf{B}_{C}$ and $\mathbf{B}_{E}$ are built similarly to the first case. The FRF of the unknown substructure $U$ is shown in Fig. 13. It can be noticed that the result is quite clean with no significant drawbacks.

To cross-check this result, a mixed interface that includes DoFs $6 z, 10 z$ and $11 z$ is used. Such DoFs represent the set intersection between DoFs $3 z, 6 z, 10 z, 11 z$ and $6 z, 9 z, 10 z, 11 z$ that provide the worst results using 4 interface DoFs. The FRF of the unknown substructure $U$ is shown in Fig. 14. It can be noticed that the result is quite bad with significant scatter around the natural frequencies and several spurious peaks.

A further check is performed using a mixed interface that includes DoFs $9 z, 10 z$ and $11 z$. DoFs $9 z, 10 z$ are both able to provide information about the rotational DoF $\theta_{y}$ (torsion of the column) but may miss the information about the rotational DoF $\theta_{x}$ (bending of the column). The FRF of the unknown substructure $U$ is shown in Fig. 15. It can be noticed that the result is very bad because the two modes around $315 \mathrm{~Hz}$ and $615 \mathrm{~Hz}$ are not clearly visible. 


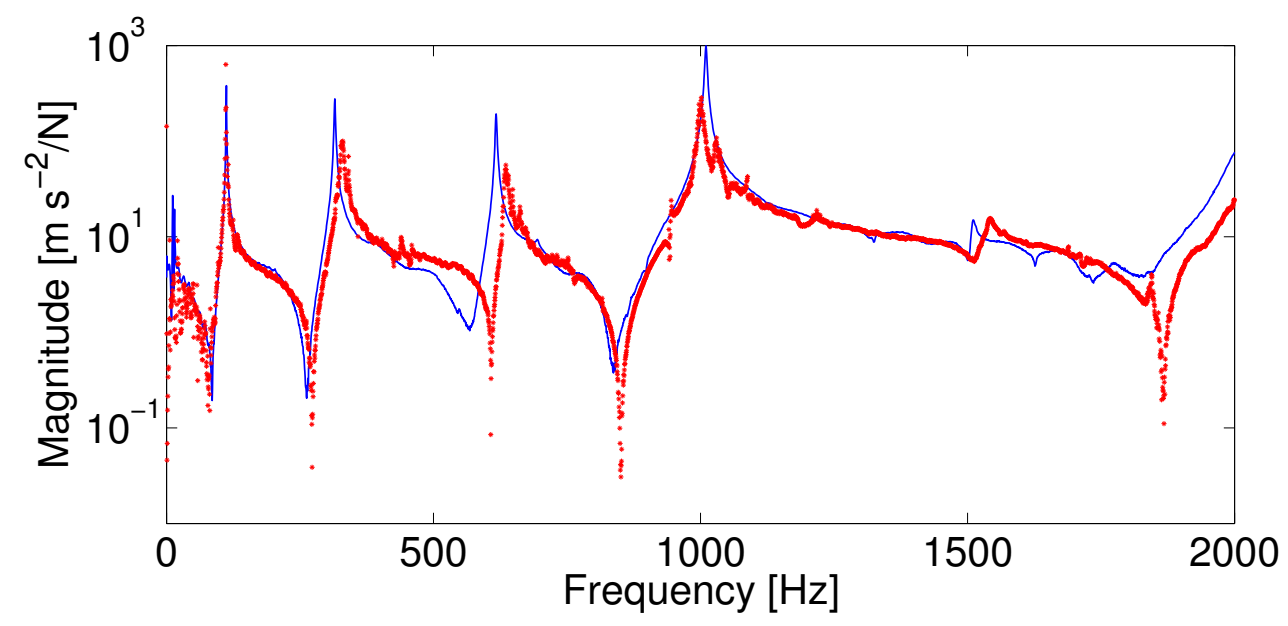

Figure 13: $H_{11 z, 11 z}^{U}$ : measured (-), computed using mixed interface with coupling DoF 11z, and internal DoFs $3 z, 9 z(* *)$.

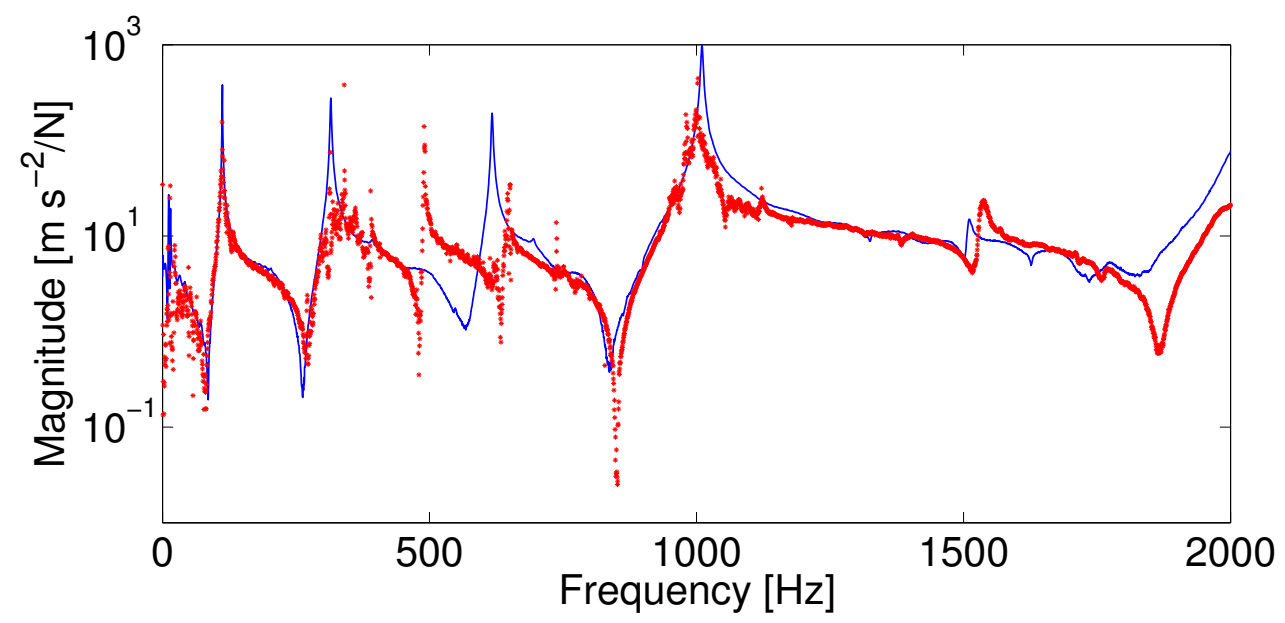

Figure 14: $H_{11 z, 11 z}^{U}$ : measured (-), computed using mixed interface with coupling DoF 11z, and internal DoFs $6 z, 10 z(* * *)$.

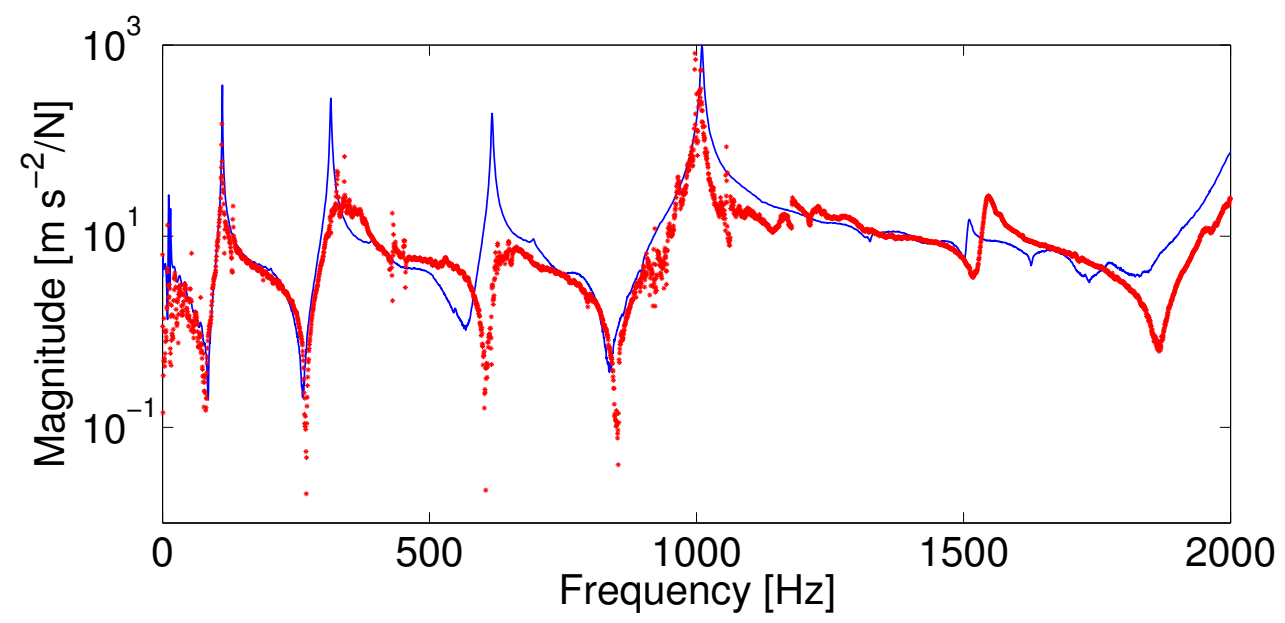

Figure 15: $H_{11 z, 11 z}^{U}$ : measured (-), computed using mixed interface with coupling DoF $11 z$, and internal DoFs $9 z, 10 z(* *)$. 


\section{CONCLUDING REMARKS}

In this paper, a procedure that ignores rotational DoFs in decoupling of substructures, connected to each other through translational and rotational DoFs, is verified on an experimental test bed. The test bed is made by a cantilever column with two staggered short arms coupled to a horizontal beam. This involves both flexural and torsional DoFs, on which rotational FRFs are quite difficult to measure. Using a mixed interface, such FRFs are neglected and substituted by FRFs involving internal translational DoFs.

Measured FRFs can be curve fitted to try to smooth out noise before using them in the decoupling procedure. However, in this case, very bad results are obtained. Therefore, raw FRFs are used. Using a mixed interface including the single translational coupling DoF and several combinations of 3 internal DoFs, the obtained results are not satisfactory in different frequency bands: in two cases results are slightly better than in other cases. Further attempts are performed using a mixed interface including the single translational coupling DoF and different combinations of 2 internal DoFs. In one case, the result is almost satisfactory, whilst in the other cases results are worse than those obtained with 3 internal DoFs. (It can be noticed that the minimum number of interface equilibrium DoFs is 3.) Therefore, increasing the number of interface DoFs to deal with an overdetermined problem does not necessarily improve the results. Conversely, decreasing the number of interface DoFs increases the variability of the results: this can lead to better results but care must be taken to avoid worse results.

\section{ACKNOWLEDGEMENTS}

This research is supported by grants from University of Rome La Sapienza and University of L'Aquila.

\section{REFERENCES}

[1] de Klerk, D., Rixen, D. J. and Voormeeren, S., General Framework for Dynamic Substructuring: History, Review, and Classification of Techniques, AIAA Journal, Vol. 46, No. 5, pp. 1169-1181, May 2008.

[2] Sestieri, A., Salvini, P. and D'Ambrogio, W., Reducing Scatter From Derived Rotational Data to Determine the Frequency Response Function of Connected Structures, Mechanical Systems and Signal Processing, Vol. 5, No. 1, pp. 25-44, Jan. 1991.

[3] Stanbridge, A. and Ewins, D., Measurement of translational and angular vibration using a scanning laser Doppler vibrometer, Shock and Vibration, Vol. 3, pp. 141-152, 1996.

[4] Bello, M., Sestieri, A., D'Ambrogio, W. and La Gala, F., Development of PZT's As Rotational Transducers, Mechanical Systems and Signal Processing, Vol. 17, No. 5, pp. 1069-1081, Sep. 2003.

[5] D'Ambrogio, W. and Fregolent, A., Promises and pitfalls of decoupling procedures, Proceedings of 26th IMAC, Orlando (U.S.A.), Feb. 2008.

[6] Sjövall, P. and Abrahamsson, T., Substructure system identification from coupled system test data, Mechanical Systems and Signal Processing, Vol. 22, No. 1, pp. 15-33, 2008.

[7] D'Ambrogio, W. and Fregolent, A., The role of interface DoFs in decoupling of substructures based on the dual domain decomposition, Mechanical Systems and Signal Processing, Vol. 24, No. 7, pp. 2035-2048, Oct. 2010, doi:10.1016/j.ymssp.2010.05.007, also in Proceedings of ISMA 2010, 1863-1880, Leuven (Belgium).

[8] D'Ambrogio, W. and Fregolent, A., Direct decoupling of substructures using primal and dual formulation, Conference Proceedings of the Society of Experimental Mechanics Series, Volume 4, Linking Models and Experiments, Vol. 2, pp. 47-76, Springer, Jacksonville, Florida USA, January 31-February 32011. 
[9] D’Ambrogio, W. and Fregolent, A., Direct Hybrid Formulation for Substructure Decoupling, Conference Proceedings of the Society for Experimental Mechanics Series, Volume 27, Topics in Experimental Dynamics Substructuring and Wind Turbine Dynamics, Vol. 2, pp. 89-107, Jacksonville, Florida USA, January 30-February 2 2012.

[10] D'Ambrogio, W. and Fregolent, A., Inverse dynamic substructuring using direct hybrid assembly in the frequency domain, Mechanical Systems and Signal Processing, Vol. 45, No. 2, pp. 360-377, 2014.

[11] Voormeeren, S. N. and Rixen, D. J., A Family of Substructure Decoupling Techniques Based on a Dual Assembly Approach, Mechanical Systems and Signal Processing, Vol. 27, pp. 379-396, Feb 2012, doi: 10.1016/j.ymssp.2011.07.028.

[12] D'Ambrogio, W. and Fregolent, A., Are Rotational DoFs Essential in Substructure Decoupling?, Dynamics of Coupled Structures, Volume 1, edited by Allen, M., Mayes, R. and Rixen, D., Conference Proceedings of the Society for Experimental Mechanics Series, pp. 27-36, Springer International Publishing, 2014. 